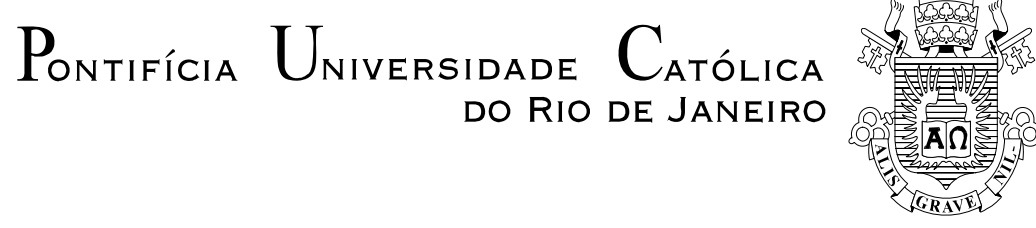

Bernardo da Rocha Spindel

Análise e Desenvolvimento de Sistema de Estimação de Modelos da Classe STAR-TREE

Dissertação de Mestrado

Dissertação apresentada como requisito parcial para obtenção do grau de Mestre pelo Programa de Pósgraduação em Engenharia Elétrica do Departamento de Engenharia Elétrica da PUC-Rio.

Orientador: Prof. Álvaro de Lima Veiga Filho

Rio de Janeiro

Agosto de 2008 


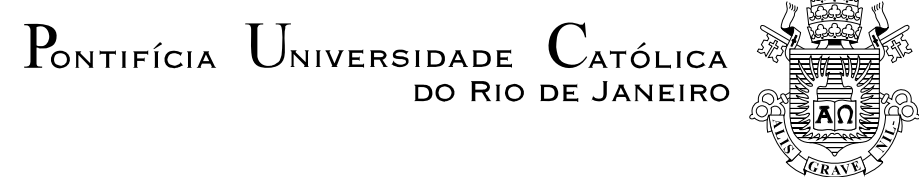

Bernardo da Rocha Spindel

\title{
Análise e Desenvolvimento de Sistema de Estimação de Modelos da Classe STAR-TREE
}

Dissertação de Mestrado apresentada como requisito parcial para obtenção do grau de Mestre pelo Programa de PósGraduação em Engenharia Elétrica do Departamento de Engenharia Elétrica do Centro Técnico Científico da PUC-Rio. Aprovada pela Comissão Examinadora abaixo assinada.

\author{
Dr. Álvaro de Lima Veiga Filho \\ Orientador \\ Departamento de Engenharia Elétrica - PUC-Rio
}

Dr. Joel Maurício Corrêa da Rosa

UFPR

Dr. Cristiano Augusto Coelho Fernandes

Departamento de Economia - PUC-Rio

Prof. José Eugenio Leal

Coordenador Setorial do Centro

Técnico Científico

Rio de Janeiro, 29 de agosto de 2008 
Todos os direitos reservados. É proibida a reprodução total ou parcial do trabalho sem autorização da universidade, do autor e do orientador.

\section{Bernardo da Rocha Spindel}

Bernardo Spindel é formado em Engenharia de Produção e mestrando em Métodos de Apoio à decisão com ênfase em estatística pela PUC-Rio. Suas áreas de interesse incluem a análise de séries temporais, métodos de regressão e estatística multivariada.

Ficha Catalográfica

\section{Spindel, Bernardo da Rocha}

Análise e desenvolvimento de sistema de estimação de modelos da classe STAR-TREE / Bernardo da Rocha Spindel ; orientador: Álvaro de Lima Veiga Filho. -2008 .

89 f. ; $30 \mathrm{~cm}$

Dissertação (Mestrado em Engenharia Elétrica) Pontifícia Universidade Católica do Rio de Janeiro, Rio de Janeiro, 2008.

Inclui bibliografia

1. Engenharia elétrica - Teses. 2. STAR-TREE. 3. Otimização. 4. Algoritmos genéticos. 5. AR. 6. Função logística. 7. Pertinência. I. Veiga Filho, Álvaro de Lima. II. Pontifícia Universidade Católica do Rio de Janeiro. Departamento de Engenharia Elétrica. III. Título. 


\section{Agradecimentos}

À minha família, pela educação, carinho e apoio, sem os quais este trabalho não poderia ter sido concluído.

Aos amigos Bruno Flach, Alexandre Street e Eduardo Faria por toda a colaboração, orientação e discussões que ao longo de todo o mestrado, me ajudaram a aprofundar meus conhecimentos.

Aos amigos Alexandre Donato, Sérgio Monteiro e Felipe Miana, que de diferentes formas e em diferentes áreas, contribuíram bastante para a realização desta dissertação.

Ao amigo Alexander Virtser, pelo incentivo e oportunidade de aprender bastante ao longo do tempo de uma pessoa de profundo conhecimento teórico e prático.

À Andréa Ribeiro Soares, por toda ajuda e incentivo dados ao longo de todo esse processo.

Ao orientador Álvaro Veiga, pelo estímulo e apoio.

À CNPq e à PUC-Rio, pelos auxílios concedidos. 


\section{Resumo}

Spindel, Bernardo da Rocha; Veiga Filho, Álvaro de Lima (Orientador). Análise e Desenvolvimento de Sistema de Estimação de Modelos da Classe STAR-TREE. Rio de Janeiro, 2008, 89p. Dissertação de Mestrado - Departamento de Engenharia Elétrica, Pontifícia Universidade Católica do Rio de Janeiro.

$\mathrm{Na}$ análise de séries temporais, os modelos lineares amplamente difundidos e utilizados, como regressões lineares e modelos auto-regressivos, não são capazes de capturar sua natureza muitas vezes não-linear,oferecendo resultados insatisfatórios. Séries financeiras, por exemplo, apresentam este tipo de comportamento. Ao longo dos últimos anos, houve o surgimento de muitos modelos não lineares para análise de séries temporais, tanto estatísticos como de inteligência computacional, baseados em redes neurais. Esta dissertação se propõe a analisar a performance do modelo STAR-Tree sob diversos cenários de conFiguração, parametrização e metodologias de estimação. Esta classe de modelos subdivide os dados de uma série temporal em regiões distintas que atendem critérios especificados em funções chamadas de pertinências. A cada região é atribuído um modelo linear auto-regressivo. Cada dado estimado pode estar em alguma das regiões com algum grau de pertinência determinado pelas funções fornecidas pelo modelo principal. Fatores como a proximidade das regiões, a suavidade das funções de pertinência e a falta de diversidade nos dados podem dificultar a estimação dos modelos. Para avaliar a qualidade das estimações sob os diversos cenários, foi construído um sistema capaz de gerar séries artificiais, importar séries externas, estimá-las sob a modelagem STARTree, e gerar simulações de Monte Carlo que avaliam a qualidade da estimação de parâmetros e a capacidade de detecção das estruturas de árvore do modelo. Ele foi utilizado como ferramenta para realizar as análises presentes na dissertação, e permitiu que se testassem diferentes conFigurações de métodos e parametrizações com facilidade.

\section{Palavras-chave}

Engenharia Elétrica, Smooth Transition Auto Regressive Tree, STARTree, Otimização, Algoritmos Genéticos, AR, Função de Logística, Pertinência. 


\section{Abstract}

Spindel, Bernardo da Rocha; Veiga Filho, Álvaro de Lima (Advisor).

Analysis and Development of a STAR-Tree Model Estimation

Software. Rio de Janeiro, 2008, 89p. MSc Dissertation - Departamento de Engenharia Elétrica, Pontifícia Universidade Católica do Rio de Janeiro.

In time series analysis, linear models that have been broadly used, such as linear regressions and auto-regressive models, are not able to capture the some times non linear nature of some data, offering poor estimation results. Financial series, for instance, show that kind of behavior. Over the last years, a great number of non linear models have been developed in order to analyze time series, some of them statistical, others based on computational intelligence techniques such as neural networks. The purpose of this dissertation is to analyze the performance of the STAR-Tree model under distinct scenarios that differ in model specification, parameterization and estimation methodologies. This class of models splits time series data into individual regions which fulfill the criteria set up by functions called pertinences. A linear model then is selected for each one of those regions. Each estimated data point can belong to one of the mentioned regions with some degree of pertinence, supplied by the above mentioned pertinence functions. Aspects like the proximity between regions, the smoothness of the pertinence functions and the lack of diversity in real data can significantly affect the estimation of models. In order to evaluate the quality of the estimations under the different proposed scenarios, a software was developed with the capabilities of generating artificial time series, importing external series, estimating them under the STAR-Tree model, and generating Monte Carlo simulations that evaluate the quality of parameter estimation and the tree structure detection capability of the model. The software was used as the single tool to generate this dissertation's analyses, and allowed that different model specifications and methods could be tested without difficulty.

\section{Keywords}

Electrical Engineering, Smooth Transition Auto Regressive Tree, STARTree, Optimization, Genetic Algorithms, AR, Logistic Function, Pertinence 


\section{Sumário}

1 INTRODUÇÃO 12

2 MODELOS DA CLASSE STAR ESTRUTURADOS EM ÁRVORE 14

2.1. Introdução 14

2.2. Formulação Geral dos Modelos STAR 14

2.3. O Modelo LSTAR 16

2.4. Árvores de Decisão 18

2.5. O Modelo STAR-Tree 22

3 ESTIMAÇÃO DE PARÂMETROS DO MRSTAR 24

3.1. Introdução $\quad 24$

3.2. Dificuldades na estimação 26

3.3. Método de Gradiente $\quad 28$

3.4. Método de Newton $\quad 30$

3.5. BFGS 32

3.6. Aplicação de Métodos de Otimização na Estimação do MRSTAR 36

3.7. Estimação por Algoritmos Genéticos 37

3.8. Resultados 40

3.8.1. Média e desvio padrão do MSE $\quad 41$

3.8.2. Pertinência média dos nós terminais $\quad 42$

3.8.3. Estimação dos parâmetros não-lineares 46

3.8.4. Estimação dos parâmetros lineares $\quad 50$

4 APLICAÇÃO DO MODELO STAR-TREE

4.1. Introdução $\quad 57$

4.2. Método CART 58

4.3. Estimação por teste de hipótese $\quad 62$

4.4. Aplicação a dados reais 68

4.4.1. Modelo ARX 70

4.4.2. Modelo STAR-Tree $\quad 72$

4.5. Conclusões 78

5 DESCRIÇÃO DO SISTEMA $\quad 80$

$\begin{array}{ll}\text { 5.1. Objetivo } & 80\end{array}$

5.2. Módulo de estimação de séries temporais 80

5.2.1. Parâmetros de estimação $\quad 81$

5.2.2. Gerador de séries artificiais 83

5.2.3. Estimação e visualização de resultados $\quad 85$

5.2.4. Simulação através de modelos gerados artificialmente 88

6 REFERÊNCIAS BIBLIOGRÁFICAS $\quad 89$ 


\section{Lista de Figuras}

$\begin{array}{lll}\text { Figura } 2.1 & \text { Função Logística } & 15\end{array}$

$\begin{array}{lll}\text { Figura } 2.2 \text { Função Exponencial } & 15\end{array}$

$\begin{array}{lll}\text { Figura } 2.3 \text { Função Degrau } & 15\end{array}$

$\begin{array}{lll}\text { Figura 2.4 Gama fixo com valor baixo, c variando } & 17\end{array}$

Figura 2.5 Gama fixo com valor alto, c variando 17

Figura 2.6 Exemplo de regiões de dados distintas com modelos específicos de estimação 18

$\begin{array}{lll}\text { Figura 2.7 } & \text { Exemplo de árvore de decisão } & 19\end{array}$

Figura 2.8 Ciclo de estimação de uma árvore de decisão 21

Figura $3.1 \quad \operatorname{LSTAR}(1)$ com $\mathrm{y}=0$ e $\mathrm{c}=0 \quad 26$

Figura $3.2 \quad \operatorname{LSTAR}(1) \operatorname{com} \mathrm{Y}=10$ e $\mathrm{c}=0 \quad 26$

Figura 3.3 Superfície da função $l(Y \mid \Phi)$ para diferentes valores de c e Y.

O modelo gerado foi um $\operatorname{LSTAR}(1)$ com $\gamma=0, c=0, B_{1}=\left[\begin{array}{ll}0 & -.8\end{array}\right], B_{2}=\left[\begin{array}{ll}0 & .6\end{array}\right]$, gerado com 1000 pontos

Figura 3.4 Superfície da função $l(Y \mid \Phi)$ para diferentes valores de c e Y.

O modelo gerado foi um LSTAR(1) com $\gamma=50, c=0, B_{1}=\left[\begin{array}{ll}0 & -.8\end{array}\right], B_{2}=\left[\begin{array}{ll}0 & .6\end{array}\right]$, gerado com 1000 pontos

Figura 3.5 Ilustração do método do Gradiente 30

Figura 3.6 Ilustração comparando o método de Newton com o do Gradiente 32

Figura 3.7 llustração do método da secante convergindo para o mínimo da função, partindo de dois pontos inicialmente selecionados

Figura 3.8 Diferentes métodos estimando o próximo passo da matriz B

Figura 3.9 Algoritmo de estimação de parâmetros por métodos de otimização

Figura 3.10 Histogramas de pertinências estimadas para um dado nó sob o método BFGS para $y=1$, $y=5$ e $y=10$

Figura 3.11 Histogramas de pertinências estimadas para um dado nó sob algoritmo genético para $y=1, y=5$ e $\gamma=10$

Figura 3.12 Dispersão dos parâmetros de localização e suavidade para $\beta_{0}=\left[\begin{array}{c}0 \\ 0.2\end{array}\right] ; \beta_{1}=\left[\begin{array}{c}0 \\ 0.9\end{array}\right]$, estimado através do método BFGS e 
$\gamma=5$

Figura 3.13 Dispersão dos parâmetros de localização e suavidade para $\beta_{0}=\left[\begin{array}{c}0 \\ -0.5\end{array}\right] ; \beta_{1}=\left[\begin{array}{c}0 \\ 0.7\end{array}\right]$, estimado através do método BFGS e $\gamma=5$

Figura 3.14 Dispersão dos parâmetros de localização e suavidade para $\beta_{0}=\left[\begin{array}{c}0 \\ 0.5 \\ -0.2\end{array}\right] ; \beta_{1}=\left[\begin{array}{c}0 \\ -0.4 \\ 0.3\end{array}\right]$, estimado através do método BFGS e $\gamma=5$

Figura 3.15 Dispersão dos parâmetros de localização e suavidade para $\beta_{0}=\left[\begin{array}{c}0 \\ 0.2\end{array}\right] ; \beta_{1}=\left[\begin{array}{c}0 \\ 0.9\end{array}\right]$, estimado através do método de Newton e $\gamma=5$

Figura 3.16 Dispersão dos parâmetros de localização e suavidade para $\beta_{0}=\left[\begin{array}{c}0 \\ -0.5\end{array}\right] ; \beta_{1}=\left[\begin{array}{c}0 \\ 0.7\end{array}\right]$, estimado através do método de Newton e $\gamma=5$

Figura 3.17 Dispersão dos parâmetros de localização e suavidade para $\beta_{0}=\left[\begin{array}{c}0 \\ 0.2\end{array}\right] ; \beta_{1}=\left[\begin{array}{c}0 \\ 0.9\end{array}\right]$, estimado através do método do Gradiente e $\gamma=5$

Figura 3.18 Dispersão dos parâmetros de localização e suavidade para $\beta_{0}=\left[\begin{array}{c}0 \\ -0.5\end{array}\right] ; \beta_{1}=\left[\begin{array}{c}0 \\ 0.7\end{array}\right]$, estimado através do método do Gradiente e $\gamma=5$

Figura 3.19 Dispersão dos parâmetros de localização e suavidade para $\beta_{0}=\left[\begin{array}{c}0 \\ 0.2\end{array}\right] ; \beta_{1}=\left[\begin{array}{c}0 \\ 0.9\end{array}\right]$, estimado através de algoritmo genético e $\gamma=5$

Figura 3.20 Dispersão dos parâmetros de localização e suavidade para $\beta_{0}=\left[\begin{array}{c}0 \\ -0.5\end{array}\right] ; \beta_{1}=\left[\begin{array}{c}0 \\ 0.7\end{array}\right], \quad$ estimado através de algoritmo genético e $\gamma=5$ 
Figura 3.21 Dispersão dos parâmetros de localização e suavidade para $\beta_{0}=\left[\begin{array}{c}0 \\ -0.5\end{array}\right] ; \beta_{1}=\left[\begin{array}{c}0 \\ 0.4\end{array}\right] ; \beta_{2}=\left[\begin{array}{c}0 \\ 0.7\end{array}\right]$, estimado através de algoritmo genético e $\gamma=5$

Figura 3.22 Estimação dos parâmetros lineares através do método BFGS para o modelo com $\beta_{0}=\left[\begin{array}{c}0 \\ -0.5\end{array}\right] ; \beta_{1}=\left[\begin{array}{c}0 \\ 0.7\end{array}\right]$ e $\gamma=5$

Figura 3.23 Valores detectados para os parâmetros lineares para cada uma das pertinências encontradas através do método BFGS para o modelo com $\beta_{0}=\left[\begin{array}{c}0 \\ -0.5\end{array}\right] ; \beta_{1}=\left[\begin{array}{c}0 \\ 0.7\end{array}\right]$ e $\gamma=5$

$\begin{array}{lll}\text { Figura 4.1 Ciclo de detecção de estrutura da árvore STAR } & 57\end{array}$

Figura 4.2 Exemplo de CART de classificação

Figura 4.3 Série de consumo médio horário de combustível, de janeiro a fevereiro de 2005

Figura 4.4 Variáveis selecionadas X série de consumo 70

Figura 4.5 Série de resíduos da estimação 71

$\begin{array}{lll}\text { Figura 4.6 Histograma dos resíduos da estimação } & 71\end{array}$

$\begin{array}{lll}\text { Figura } 4.7 & \text { Probabilidade empírica acumulada dos resíduos } & 72\end{array}$

$\begin{array}{lll}\text { Figura 4.8 Árvore estimada pelo modelo STAR-Tree } & 73\end{array}$

Figura 4.9 Pertinência acumulada capturada pelos nós de decisão a cada dado da série $\quad 74$

Figura 4.10 Funções de pertinência resultantes da estimação 75

Figura 4.11 Série de resíduos da estimação do modelo STAR 76

Figura 4.12 Histograma dos resíduos da estimação 76

$\begin{array}{lll}\text { Figura 4.13 Probabilidade empírica acumulada dos resíduos } & 77\end{array}$

$\begin{array}{lll}\text { Figura 4.14 Estimação do modelo STAR-Tree } & 78\end{array}$

Figura 5.1 Escolha do método de estimação dos parâmetros não lineares 81

Figura 5.2 Exemplo de parametrização para algoritmos genéticos 82

Figura 5.3 Escolha do método de crescimento da árvore 83

Figura 5.4 Escolha dos parâmetros de um nó de decisão 84

Figura 5.5 Escolha dos parâmetros de um nó de terminal 84

Figura 5.6 Geração de séries a partir de modelos previamente montados 95

Figura 5.7 Processo de estimação da série $\quad 86$

Figura 5.8 Exemplo de estimação de série 86

Figura 5.9 Funções de pertinência nos modelos gerador e estimado $\quad 87$

Figura 5.10 Análise dos resíduos decorrentes da estimação 87

Figura 5.11 Exemplo de relatório gerado pelo módulo de simulações 88 


\section{Lista de Tabelas}

Tabela 4.1 Descrição das estatísticas de avaliação dos modelos 69

$\begin{array}{lll}\text { Tabela } 4.2 & \text { Parâmetros resultantes da estimação } & 70\end{array}$

$\begin{array}{lll}\text { Tabela 4.3 Estatísticas de avaliação do modelo ARX } & 72\end{array}$

Tabela 4.4 Parâmetros lineares resultantes da estimação do modelo STARTree $\quad 75$

$\begin{array}{lll}\text { Tabela } 4.5 & \text { Estatísticas de avaliação do modelo STAR-Tree }\end{array}$ 